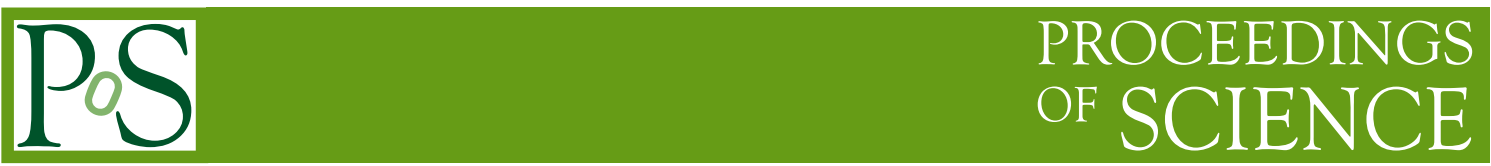

\title{
On the origin of $\mathrm{Y}(4260)$ and the $J^{P C}=1^{--}$exotic mesons
}

\author{
Sachiko Takeuchi ${ }^{* \dagger}$ \\ Japan College of Social Work, Kiyose, Tokyo 204-8555, Japan \\ Research Center for Nuclear Physics (RCNP), Osaka Univ., Ibaraki, Osaka, 567-0047, Japan \\ RIKEN Nishina Center, Wako, Saitama 351-0198, Japan \\ E-mail: S.takeuchidjcsw.ac.jp
}

\section{Makoto Takizawa}

Showa Pharmaceutical University, Machida, Tokyo 194-8543, Japan

RIKEN Nishina Center, Wako, Saitama 351-0198, Japan

J-PARC Branch, KEK Theory Center, IPNS, KEK, Tokai, Ibaraki, 319-1106, Japan

E-mail: takizawadac.shoyaku.ac.jp

\begin{abstract}
The $q \bar{q} s \bar{s}$ and $q \bar{q} c \bar{c} J^{P C}=1^{--}$systems are investigated by a quark hadron hybrid model, where the 14 relevant two-meson channels are coupled, while the quark degrees of freedom appear in the short range region. In each of the $q \bar{q} s \bar{s}$ and $q \bar{q} c \bar{c}$ systems, one or more poles have been found. For the $q \bar{q} c \bar{c}$, a pole with a very narrow width appears very close to the $\omega \chi_{c 1}$ threshold, which is also close to the $\bar{D} D_{1}$ and $\bar{D} D_{1}^{\prime}$ thresholds. There are two poles in the $q \bar{q} s \bar{s}$, both of which have a rather larger width. We argue that they can be seeds of the observed exotic mesons like the $Y(4260)$.
\end{abstract}

XIII Quark Confinement and the Hadron Spectrum - Confinement 2018

31 July - 6 August 2018

Maynooth University, Ireland

\footnotetext{
*Speaker.

${ }^{\dagger}$ A part of this work is reported in ref. [四]. This work was supported in part by JSPS KAKENHI No. 16K05361.
} 


\section{Introduction}

In 2003, Belle collaboration reported that they had found a very narrow peak in the decay of $B^{ \pm}$to $J / \psi \pi^{+} \pi^{-} K^{ \pm}$, which is now called $X(3872)$ [[]]. It was confirmed also by other types of reactions [B]]. In 2005, BaBar collaboration reported that there was a broad resonance in the initial state radiation (ISR) process of $e^{+} e^{-}$collision experiments with the final $\pi^{+} \pi^{-} J / \psi$ channel [四]. This resonance, called $Y(4260)$ (or $\psi(4260)$ ), has been investigated mainly by ISR also by other

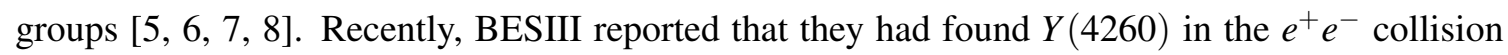
without ISR and that the resonance has a lighter mass, $4222.0 \pm 3.1 \mathrm{MeV}$, with a width of $44.1 \pm 4.3$ $\mathrm{MeV}$ [Q]. Though the reported values of the masses seem not fully agreed with each other yet, the existence of $Y(4260)$ seems to be now established. BESIII also reported another broad resonance at around $4320 \mathrm{MeV}$ [Q]. A charged charmoniumlike peak, $Z_{c}(3900)$, was found in the decay of $Y(4260)$ [ [8, [0]. Recently LHCb collaboration reported that they had found two resonances, $P_{c}(4380)^{+}$and $P_{c}(4450)^{+}$, which are considered to be $u u d c \bar{c}$ states in the $\Lambda_{b}$ decay [प]]. The hadrons with the $c \bar{c}$ component seem to have a rich mass spectrum in this energy region.

There are a few candidates of non $q \bar{q}$ mesons also among the unflavored mesons [B]]. For example, $f_{0}(980)$ is considered to have a large amount of $s \bar{s} q \bar{q}$ component because their decay branching ratio to the final $K \bar{K}$ is large even though their masses are very close to the $K \bar{K}$ threshold. There are more observed states than those of a simple $q \bar{q}$ model predicts in the higher energy region, though many of the observed states are not established yet and are still required to be confirmed.

The multiquark approach can be a strong tool to investigate such exotic states: the properties of $X(3872)$ can be explained by a $1^{++} q \bar{q} c \bar{c}$ configuration which couples to the $\chi_{c 1}(2 P)$ as well as the two-meson states [2], [3]]. Or, it is found that the color octet $q^{3}$ in the $u u d c \bar{c}$ configuration gives rise to a resonance around $\Sigma_{c}^{(*)} \bar{D}^{*}$ threshold, which may contribute to the LHCb pentaquarks, $P_{c}$ 's [प]4].

Here we investigate the $J^{P C}=1^{--} q \bar{q} Q \bar{Q}$ systems, employing a quark hadron hybrid model. In this model, the asymptotic states are two mesons, where the quark degrees of freedom are hidden in the mesons. In the short-range region, where the two mesons are close to each other, the quark degrees of freedom appears as the rearrangement occurs from $q \bar{q}-Q \bar{Q}$ to $q \bar{Q}-Q \bar{q}$ systems. These quark effects can be included in the model as a hadron interaction, which enables us to solve complicated systems. As we will show later, we have found this model can produce resonance(s) for the $J^{P C}=1^{--} q \bar{q} c \bar{c}$ or $q \bar{q} s \bar{s}$ systems. We argue that they can be a seed of the observed exotic mesons like $Y(4260)$.

\section{Model}

\section{$2.1 q \bar{q} Q \bar{Q}$ classification}

First we classify the $q \bar{q} Q \bar{Q} J^{P C}=1^{- \pm}$systems with the orbital $(0 s)^{2} 0 p$ configuration by mapping them onto two-meson states. The two-meson states we consider here consist of the $L=0$ and 1 quark-antiquark mesons: those of ${ }^{1} S_{0}\left(J^{P C}=0^{-+}\right),{ }^{3} S_{1}\left(1^{--}\right),{ }^{1} P_{1}\left(1^{+-}\right)$, and ${ }^{3} P_{J}\left(J^{++}\right)$. Combining the $q \bar{q}$ and the $Q \bar{Q}$ states of the above quantum numbers, one can make ten $q \bar{q} Q \bar{Q}(0 s)^{2} 0 p 1^{--}$ states and eight $1^{-+}$states as listed in Table $\mathbb{~}$. 
There are two independent color configurations for the totally color-singlet $q \bar{q} Q \bar{Q}$ system. One is the configuration in which both of the $q \bar{q}$ and $Q \bar{Q}$ are color-singlet, which we denote $(q \bar{q})_{1}(Q \bar{Q})_{1}$. The other is the one where both of them are color-octet, $(q \bar{q})_{8}(Q \bar{Q})_{8}$. The former can be mapped onto the two-meson states directly, but the latter cannot. It is necessary to apply a quark rearrangement in the color spin orbital space in order to map it onto the two-meson states:

$$
(q \bar{q})_{8}(Q \bar{Q})_{8}=\sqrt{\frac{9}{8}} T^{-1}(q \bar{Q})_{1}(Q \bar{q})_{1}-\sqrt{\frac{1}{8}}(q \bar{q})_{1}(Q \bar{Q})_{1},
$$

where $T$ is the transfer matrix of the ten (eight) channels in the spin orbital space. The $(q \bar{Q})_{1}(Q \bar{q})_{1}$ 's correspond to $\bar{D} D$ states when $Q$ is taken to be the charm quark. For the $1^{--}$states, they are:

$$
\begin{aligned}
& {\left[\bar{D} D_{1}\right]_{-},\left[\bar{D} D_{1}^{\prime}\right]_{+},\left[\bar{D}^{*} D_{0}\right]_{+},\left[\bar{D}^{*} D_{1}\right]_{-},\left[\bar{D}^{*} D_{1}^{\prime}\right]_{+},\left[\bar{D}^{*} D_{2}\right]_{+} \text {for relative } S \text { wave }} \\
& \bar{D} D,\left[\bar{D} D^{*}\right]_{-},\left.\left(\bar{D}^{*} D^{*}\right)\right|_{S=0,2} \text { for relative } P \text { wave }
\end{aligned}
$$

where $[\bar{A} B]_{ \pm}$stands for $(\bar{A} B \pm \bar{B} A) / \sqrt{2}$. In summary, the $q \bar{q} Q \bar{Q}$ states can be classified into $201^{--}$ two-meson states and $161^{-+}$states all together. Note that, suppose one of the $(q \bar{q})_{8}(Q \bar{Q})_{8}$ states plays an important role, then it is necessary to take many two-meson channels into account in order to see the effects. Let us point out also that these $201^{--}$two-meson states are independent but not orthogonal to each other as seen from eq. (‥J). The $\bar{D} D_{1}$ state is orthogonal to the $\bar{D} D_{1}^{\prime}$, for example. The $\bar{D} D_{1}$ and $\omega \chi_{c 1}$ states, for example, however, are independent but not orthogonal to each other due to the quark degrees of freedom.

\section{2 quark hadron hybrid model}

We employ a quark Hamiltonian which has the kinetic term and the two-body interaction terms: the central, the spin-spin, the spin-orbit, and the tensor terms. They are considered to come from the confinement force and one-gluon exchange force as those in the conventional quark model. We assume that all the interaction terms of the present model have the color factor, $\lambda \cdot \lambda$. Since we only consider the quark degrees of freedom within the orbital $(0 s)^{2} 0 p$ configuration, it is enough to determine the size of the matrix elements of the interaction with respect to the $0 s$ or $0 p$

Table 1: $q \bar{q} Q \bar{Q}$ spin flavor orbital classification. $S$ is the meson spin, and $L_{r}$ is the relative meson orbital angular momentum. The meson names are those when $Q$ and $\bar{Q}$ are taken to be the charm quark and antiquark.

\begin{tabular}{cccclll}
\hline$q \bar{q}$ & $Q \bar{Q}$ & $S$ & $L_{r}$ & $J^{P C}$ & mesons & $q \bar{q} \leftrightarrow Q \bar{Q}$ \\
\hline${ }^{1} S_{0}$ & ${ }^{1} P_{1}$ & 1 & $0 s$ & $1^{--}$ & $\eta h_{c 1}$ & $h_{1} \eta_{c}$ \\
${ }^{3} S_{1}$ & ${ }^{3} P_{J}$ & 1 & $0 s$ & $1^{--}$ & $\omega \chi_{c J}$ & $f_{J} J / \psi$ \\
${ }^{1} S_{0}$ & ${ }^{3} S_{1}$ & 1 & $0 p$ & $1^{--}$ & $\eta J / \psi$ & $\omega \eta_{c}$ \\
\hline${ }^{1} S_{0}$ & ${ }^{3} P_{1}$ & 1 & $0 s$ & $1^{-+}$ & $\eta \chi_{c 1}$ & $f_{1} \eta_{c}$ \\
${ }^{3} S_{1}$ & ${ }^{1} P_{1}$ & 1 & $0 s$ & $1^{-+}$ & $\omega h_{c 1}$ & $h_{1} J / \psi$ \\
${ }^{1} S_{0}$ & ${ }^{1} S_{0}$ & 0 & $0 p$ & $1^{-+}$ & $\eta \eta_{c}$ & - \\
${ }^{3} S_{1}$ & ${ }^{3} S_{1}$ & $0,1,2$ & $0 p$ & $1^{-+}$ & $\omega J / \psi$ & - \\
\hline
\end{tabular}


configurations; we do not have to assume the potential function shape in the orbital space. So, let us just consider the matrix elements of the quark Hamiltonian with respect to the $0 \ell q \bar{q}$ or $q q$ state:

$$
\left\langle H_{q}\right\rangle=\sum_{i}\left(m_{q}+\left\langle K_{q}\right\rangle\right)+\sum_{i<j} \lambda_{i} \cdot \lambda_{j}\left(c_{0 \ell}^{c}+c_{0 \ell}^{\sigma \sigma} \sigma_{i} \cdot \sigma_{j}+c_{0 \ell}^{L S} \mathscr{O}_{i j}^{L S}+c_{0 \ell}^{A L S} \mathscr{O}_{i j}^{A L S}+c_{0 \ell}^{T} \mathscr{O}_{i j}^{T}\right),
$$

where $c_{0} \ell^{\prime}$ s are the matrix elements, and $\mathscr{O}$ 's are the noncentral operators of the quarks. We obtain the $c$ 's from the hadron mass spectra assuming that the orbital part of the $q \bar{q}$ mesons or $q^{3}$ baryons can also be approximated by the $0 s$ or $0 p$ configuration of the same size parameters as those in the above $(0 s)^{2} 0 p$ configuration. This assumption is valid when one takes the size parameter to be $b_{\text {red }}=\sqrt{x_{0}^{2} / m_{\text {red }}}$, where $x_{0}$ is a constant $\left(\sim 0.6 \mathrm{fm}^{1 / 2}\right)$ and $m_{\text {red }}$ is the reduced mass of the relevant quarks [U4]. The $b_{\text {red }}$ between $u \bar{u}, c \bar{u}$, or $c \bar{c}$ is $0.69,0.53$, or $0.29 \mathrm{fm}$, respectively.

The matrix elements for the interaction between $c$ and $\bar{c}, c_{0 \ell}^{\mathscr{Q}}(c \bar{c})$, can be determined from the $c \bar{c}$ meson masses straightforwardly. We use not all the light hadron masses as they are, however, in order to determine the $c$ 's because some of the light mesons are not regarded as a simple $q \bar{q}$ meson. For the $s \bar{s}\left({ }^{1} S_{0}\right)$ mass, we use the mass of $\sqrt{m_{K}^{2}-m_{\pi}^{2}}=476.03 \mathrm{MeV}$ instead of the observed $\eta$ or $\eta^{\prime}$ masses. We take $\left(m_{\omega}+\frac{64}{3} c_{0 s}^{\sigma \sigma}(u u)\right)$ for the $u \bar{u}\left({ }^{1} S_{0}\right)$ mass with $c_{0 s}^{\sigma \sigma}(u u)=-\frac{1}{32}\left(2 m_{\Sigma_{c}^{*}}+m_{\Sigma_{c}}-\right.$ $\left.3 m_{\Lambda_{c}}\right)=-19.70 \mathrm{MeV}$. As for the $q c$ or $q s$ interaction, we use the same $c$ 's for the ones between $q \bar{c}$ or $q \bar{s}$ except for the $c_{0 s}^{\sigma \sigma}$, which we obtain from the baryon mass spectra: we use $c_{0 s}^{\sigma \sigma}(u s)=$ $-\frac{1}{16}\left(m_{\Sigma^{*}}-m_{\Sigma}\right)=-11.96 \mathrm{MeV}$. Using $-\frac{3}{32}\left(2 m_{\Xi_{c}^{*}}-m_{\Xi_{c}^{\prime}}-m_{\Xi_{c}}\right)=c_{0 s}^{\sigma \sigma}(u s)+c_{0 s}^{\sigma \sigma}(u c)+c_{0 s}^{\sigma \sigma}(s c)$ and assuming $c_{0 s}^{\sigma \sigma}(u c)=c_{0 s}^{\sigma \sigma}(s c)$, we have $c_{0 s}^{\sigma \sigma}(u c)=-5.47 \mathrm{MeV}$. Moreover, we assume that $D_{1}(2420)$ $\left[D_{1}^{\prime}(2430)\right]$ corresponds to the $c \bar{q}$ state where the light quark spin with the angular momentum, $j_{\bar{q}}=s_{\bar{q}}+\ell$, is $\frac{3}{2}\left[\frac{1}{2}\right]$. Those of $q \bar{s}$, we assume that $K_{1}(1270)\left[K_{1}(1400)\right]$ corresponds to that of $\frac{3}{2}$ $\left[\frac{1}{2}\right]$.

Now let us define the two-meson interaction arising from the quark degrees of freedom, by taking the matrix elements of the quark Hamiltonian with respect to the the quark configuration, $(q \bar{q})_{1}(Q \bar{Q})_{1}$. Because we assume the quark interaction has the color factor, the diagonal part of the Hamiltonian should be free:

$$
\left\langle(q \bar{q})_{1}(Q \bar{Q})_{1}(\alpha) ;(0 s)^{2} 0 p\left|\left(H_{q}-E\right)\right|(q \bar{q})_{1}(Q \bar{Q})_{1}\left(\alpha^{\prime}\right) ;(0 s)^{2} 0 p\right\rangle=\left(M_{1}^{\alpha}+M_{2}^{\alpha}+K_{0 \ell}^{\alpha}-E\right) \delta_{\alpha \alpha^{\prime}}
$$

where $\alpha$ is the quantum number of the four-quark state. The $M_{1}^{\alpha}$ and $M_{2}^{\alpha}$ are the mass of each of the two mesons in the $\alpha$ channel as listed in Table $\square$. The $K_{0 \ell}^{\alpha}$ term comes from the relative meson kinetic energy, which has the $0 s$ or $0 p$ configuration, and becomes $\frac{3}{4 x_{0}^{2}}$ or $\frac{5}{4 x_{0}^{2}}$, respectively.

With the same procedure we have for the $\bar{D} D$

$$
\left\langle(q \bar{Q})_{1}(Q \bar{q})_{1}(\beta) ;(0 s)^{2} 0 p\left|\left(H_{q}-E\right)\right|(q \bar{Q})_{1}(Q \bar{q})_{1}\left(\beta^{\prime}\right) ;(0 s)^{2} 0 p\right\rangle=\left(M_{1}^{\beta}+M_{2}^{\beta}+K_{0 \ell}^{\beta}-E\right) \delta_{\beta \beta^{\prime}}
$$

Nonzero values appear in the off-diagonal part where the rearrangement of quarks occurs, from which we define the two-meson potential as:

$$
\left\langle(q \bar{q})_{1}(Q \bar{Q})_{1}(\alpha) ;(0 s)^{2} 0 p\left|\left(H_{q}-E\right)\right|(q \bar{Q})_{1}(Q \bar{q})_{1}(\beta) ;(0 s)^{2} 0 p\right\rangle=V_{\alpha \beta}(E)
$$

The potential $V_{\alpha \beta}(E)$ depends on the energy because the overlapping term which proportional to $E$ survives due to the rearrangement. 
Table 2: Meson masses used for the potential (in MeV). Data are taken from ref. [B]].

\begin{tabular}{cccccc}
\hline $0^{-+}\left({ }^{1} S_{0}\right)$ & $1^{--}\left({ }^{3} S_{1}\right)$ & $1^{+-}\left({ }^{1} P_{1}\right)$ & $0^{++}\left({ }^{3} P_{0}\right)$ & $1^{++}\left({ }^{3} P_{1}\right)$ & $2^{++}\left({ }^{3} P_{2}\right)$ \\
\hline$u \bar{u}\left({ }^{1} S_{0}\right)$ & $\omega$ & $h_{1}(1170)$ & $f_{0}(980)$ & $f_{1}(1285)$ & $f_{2}(1270)$ \\
362.37 & 782.65 & 1170 & 990 & 1281.9 & 1275.5 \\
$s \bar{s}\left({ }^{1} S_{0}\right)$ & $\phi(1020)$ & $h_{1}(1380)$ & $f_{0}(1500)$ & $f_{1}(1420)$ & $f_{2}^{\prime}(1525)$ \\
476.03 & 1019.461 & 1407 & 1504 & 1426.4 & 1525 \\
$\eta_{c}$ & $J / \psi$ & $h_{c}$ & $\chi_{c 0}$ & $\chi_{c 1}$ & $\chi_{c 2}$ \\
2983.9 & 3096.900 & 3525.38 & 3414.71 & 3510.67 & 3556.17 \\
\hline $0^{-}\left({ }^{1} S_{0}\right)$ & $1^{-}\left({ }^{3} S_{1}\right)$ & $0^{+}\left({ }^{3} P_{0}\right)$ & $1^{+}\left(j_{\bar{q}}=\frac{1}{2}\right)$ & $1^{+}\left(j_{\bar{q}}=\frac{3}{2}\right)$ & $2^{+}\left({ }^{3} P_{2}\right)$ \\
\hline$K$ & $K^{*}$ & $K_{0}^{*}(1430)$ & $K_{1}(1400)$ & $K_{1}(1270)$ & $K_{2}^{*}(1430)$ \\
495.644 & 891.66 & 1425 & 1403 & 1272 & 1429 \\
$D$ & $D^{*}$ & $D_{0}^{*}(2400)$ & $D_{1}(2430)$ & $D_{1}(2420)$ & $D_{2}^{*}(2460)$ \\
1869.65 & 2008.56 & 2318 & 2427 & 2423.2 & 2463.1 \\
\hline
\end{tabular}

So, we have the Hamiltonian for the two meson systems as:

$$
H_{h}^{\alpha \beta}=\left(M_{1}^{\alpha}+M_{2}^{\alpha}+K^{\alpha}\right) \delta_{\alpha \beta}+|0 \ell\rangle V_{\alpha \beta}(E)\langle 0 \ell|
$$

This Hamiltonian can be used also for the long range region, where the system is free. There is no interaction among the $c \bar{c}-q \bar{q}$ channels, nor among the $\bar{D} D$ channels. The Hamiltonian has the interaction of a range of the hadron size, which appears only between the $c \bar{c}-q \bar{q}$ and the $\bar{D} D$ channels. In this work, we further restrict ourselves to use the channels whose relative orbital momentum is $S$-wave: we take the channels where $0 \ell=0 s$ in eq. ([.]). There are 14 such channels for the $1^{--}$states out of 20 . The above meson interaction becomes a simple gaussian separable potential, which enables us to solve the many-channel coupled systems rather easily.

Let us remark that we replace the reduced mass in the denominator with that of the real meson masses in the kinetic term of the above equation, $K^{\alpha}$, in order to make the kinematics of the system realistic. Let us also note that we ignore the kinetic term which operates over the rearrangement part of the normalization in eq. ([.]). This term produces, e.g., a strong repulsion when the system has a Pauli-forbidden state(s). In the present work on the $q \bar{q} Q \bar{Q}$ systems there is no such strong repulsion, and the term appears only in the off-diagonal channels, which we ignore because it is rather small.

\section{Results and discussion}

We employ the complex scaling method to solve the systems [니]. It is easier to employ the method to search poles in the $S$-matrix when many channels are coupled. We confirmed existence of these poles and their energies also by solving the Lippmann Schwinger equation.

In the $c \bar{c} q \bar{q} 1^{--}$systems, we have found a pole with an energy of $4293.37-0.23 i \mathrm{MeV}$. The width of this resonance is very small, $0.5 \mathrm{MeV}$, and the real part of the energy is very close to the two-meson thresholds: the $\bar{D}^{+} D_{1}^{-}$threshold, $4292.85 \mathrm{MeV}$, the $\omega \chi_{c 1}, 4293.32 \mathrm{MeV}$, and the 
Table 3: The energy and the width of the resonance poles (in $\mathrm{MeV}$ ). The $c \bar{c} q \bar{q} 1^{--}$system has one pole while the $s \bar{s} q \bar{q} 1^{--}$system has two poles.

\begin{tabular}{lrr}
\hline system & energy & width \\
\hline$c \bar{c} q \bar{q} 1^{--}$ & 4293.37 & 0.46 \\
\hline$s \bar{s} q \bar{q} 1^{--}$ & 2160.54 & 20.08 \\
& 2288.32 & 67.86 \\
\hline
\end{tabular}

$\bar{D}^{+} D_{1}^{\prime-}, 4296.65 \mathrm{MeV}$. Since the resonance is close to the thresholds, the components of this resonance are mostly these three two-meson states. In the short range region, however, the size of both of the $f_{1} J / \psi$ and $f_{2} J / \psi$ components become comparable to the above three states. Modes which consist of many two-meson states seem important here, though more investigation is required. The resonance does not correspond directly to the observed $Y(4260)$, because it requires an additional attraction to reduce the mass by about $90 \mathrm{MeV}$. It, however, strongly suggests that this resonance, or the quark rearrangement, induces the exotic mesons like $Y(4260)$.

In the $s \bar{s} q \bar{q} 1^{--}$systems, we have found two poles as listed in Table 3 . The observed $\phi(2160)$ may have a component of the lower one at $2160 \mathrm{MeV}$, whose component mainly consists of $\bar{K}^{*} K_{1}(1270)$ and $\left(\bar{K}^{*}\right)_{8}\left(K_{1}(1270)\right)_{8}$; the latter corresponds to the superposition of the $\omega f_{0}(1500)$, $f_{2}^{\prime}(1525) \phi$, and many other two-meson states. There are no established $1^{--}$resonances which are close to the higher resonance. It has a large width and consists of many components such as $K^{*} K_{1}(1400), \omega f_{2}^{\prime}(1525)$ and several other states. There is one more state at around $2360-40 i$ $\mathrm{MeV}$ which appear as an energy eigenstate in the complex scaling method. It, however, seems not to be a resonance according to the Lippmann Schwinger calculation though the scattering observables vary rapidly around this energy.

There is a model ambiguities which comes from the meson assignments, or level mixing of the mesons, in the process of obtaining the matrix elements $c$ 's. The decay widths of some of the mesons which construct the two-meson states, e.g., the $D_{1}$ or $D_{1}^{\prime}$ mesons, are large, and should be included when one performs more realistic calculation. Moreover, it is probably necessary to include the pion exchange effects in the $\bar{D} D$ channels. It is also interesting to see the effects from the coupling to the $c \bar{c}$ mesons, such as $\psi(3 S)$ or $\psi(4 S)$, with an annihilation of the light quark pair.

In the present work, we do not actually use the fact that the isospin of the system is 0 , when we derive the potential $V_{\alpha \beta}(E)$. The rearrangement can be done similarly for, e.g. $u \bar{d} c \bar{c}$ systems. Let us point out that the above poles can appear even for the charged systems in similar energies except for the effects of the meson exchange or those of the coupling to the $c \bar{c}$ or $s \bar{s}$ state.

\section{Summary}

In this work we investigate the $q \bar{q} s \bar{s}$ and $q \bar{q} c \bar{c} J^{P C}=1^{--}$systems by employing a quark hadron hybrid model. We introduce the 14 relevant two-meson channels, whose short range includes the quark degrees of freedom. For the $q \bar{q} c \bar{c}$, a pole appears just around the $\omega \chi_{c 1}, \bar{D} D_{1}$, and $\bar{D} D_{1}^{\prime}$ thresholds with a very narrow width. There are two poles found in the $q \bar{q} s \bar{s}$, each of which has 
a rather larger width. The results strongly suggest that the quark degrees of freedom contribute largely to form the observed exotic mesons like $Y(4260)$.

\section{References}

[1] S. Takeuchi and M. Takizawa, PoS Hadron 2017, 109 (2018).

[2] S. K. Choi et al. [Belle Collaboration], Phys. Rev. Lett. 91, 262001 (2003)

[3] M. Tanabashi et al. [Particle Data Group], Phys. Rev. D 98, no. 3, 030001 (2018).

[4] B. Aubert et al. [BaBar Collaboration], Phys. Rev. Lett. 95, 142001 (2005);

[5] Q. He et al. [CLEO Collaboration], Phys. Rev. D 74, 091104 (2006)

[6] C. Z. Yuan et al. [Belle Collaboration], Phys. Rev. Lett. 99, 182004 (2007);

[7] J. P. Lees et al. [BaBar Collaboration], Phys. Rev. D 86, 051102 (2012)

[8] M. Ablikim et al. [BESIII Collaboration], Phys. Rev. Lett. 110, 252001 (2013)

[9] M. Ablikim et al. [BESIII Collaboration], Phys. Rev. Lett. 118, no. 9, 092001 (2017)

[10] Z. Q. Liu et al. [Belle Collaboration], Phys. Rev. Lett. 110, 252002 (2013)

[11] R. Aaij et al. [LHCb Collaboration], Phys. Rev. Lett. 115, 072001 (2015).

[12] M. Takizawa and S. Takeuchi, Prog. Theor. Exp. Phys. 2013, 0903 D01 (2013).

[13] S. Takeuchi, K. Shimizu and M. Takizawa, Prog. Theor. Exp. Phys. 2014 123D01(2014) Erratum: [ Prog. Theor. Exp. Phys. 2015 079203(2015)]

[14] S. Takeuchi and M. Takizawa, Phys. Lett. B 764, 254 (2017).

[15] T. Myo, Y. Kikuchi, H. Masui and K. Katō, Prog. Part. Nucl. Phys. 79, 1 (2014). 